\title{
Proteomic analysis of the shistosome tegument and its surface membranes
}

\author{
Simon Braschi ${ }^{+}$, William Castro Borges, R Alan Wilson \\ Department of Biology, University of York, York YO10 5DD, United Kingdom
}

\begin{abstract}
The tegument surface of the adult schistosome, bounded by a normal plasma membrane overlain by a secreted membranocalyx, holds the key to understanding how schistosomes evade host immune responses. Recent advances in mass spectrometry (MS), and the sequencing of the Schistosoma mansoni transcriptome/genome, have facilitated schistosome proteomics. We detached the tegument from the worm body and enriched its surface membranes by differential extraction, before subjecting the preparation to liquid chromatography-based proteomics to identify its constituents. The most exposed proteins on live worms were labelled with impearmeant biotinylation reagents, and we also developed methods to isolate the membranocalyx for analysis. We identified transporters for sugars, amino acids, inorganic ions and water, which confirm the importance of the tegument plasma membrane in nutrient acquisition and solute balance. Enzymes, including phosphohydrolases, esterases and carbonic anhydrase were located with their catalytic domains external to the plasma membrane, while five tetraspanins, annexin and dysferlin were implicated in membrane architecture. In contrast, few parasite proteins could be assigned to the membranocalyx but mouse immune response proteins, including three immunoglobulins and two complement factors, were detected, plus host membrane proteins such as CD44, integrin and a complement regulatory protein, testifying to the acquisitive properties of the secreted bilayer.
\end{abstract}

Key words: Schistosoma mansoni - membrane proteins - mass spectrometry - membranocalyx - immune evasion

Following infection of the mammalian host by skin penetration, schistosomula of Schistosoma mansoni enter the vasculature and migrate to the hepatic portal system where they mature and begin egg deposition. In this potentially hostile environment the parasites are constantly exposed to host immune responses, yet their ability to thrive for several decades (Harris et al. 1984) testifies to the possession of effective evasion mechanisms. Unlike nematodes, which are protected by a cuticle, schistosomes are covered by a living syncytium, called the tegument (Figure). This tissue is bounded at its basal surface by a conventional invaginated plasma membrane, whilst its apical surface has an unusual heptalaminate appearance (Hockley \& McLaren 1973). This latter structure was interpreted as a normal plasma membrane overlain by a membrane-like secretion, termed the membranocalyx (Wilson \& Barnes 1974b). Nucleated cell bodies ("cytons") are situated beneath the muscle layers, connected to the tegument by microtubule-lined cytoplasmic channels (Figure). Protein synthesising machinery and the Golgi apparatus are located exclusively in the cell body. Two forms of secretory inclusions, discoid bodies and multilaminate vesicles, are produced in the cell bodies and travel to the tegument via the cytoplasmic con-

Financial support: The WHO/UNDP/World Bank Programme for Research and Training in Tropical Diseases, The Biotechnology and Biological Sciences Research Council

${ }^{+}$Corresponding author: simon.braschi@googlemail.com

Received 25 May 2006

Accepted 26 June 2006 nections. The discoid bodies contain carbohydrate-rich material and are thought to contribute to the tegument ground substance (Wilson \& Barnes 1974a). The multilaminate vesicles contain whirls of tightly packed membrane-like material, and are formed when small transport vesicles from the Golgi apparatus fuse with vacuoles in the cell body (Wilson \& Barnes 1974a). Within the lumen of these vacuoles, membrane-like bilayers self-assemble to create the characteristic multilaminate appearance (Figure). In the syncytium, multilaminate vesicles have been observed at the base of tegumental pits (Figure), where their bounding membranes appear to fuse with the apical plasma membrane (Wilson \& Barnes 1974a), releasing their cargo that unravels and diffuses laterally to form the secreted membranocalyx (Wilson \& Barnes 1977). This model of the tegument surface envisages the secreted membranocalyx as a physical barrier to immune attack, protecting the underlying, vulnerable plasma membrane. Determining the composition and structural organization of these surface bilayers is therefore crucial for an understanding of immune evasion by schistosomes.

Methods were developed more than 20 years ago to detach the tegument using detergents (Kusel 1970, Oaks et al. 1981, McDiarmid et al. 1983), chemicals (Bennett \& Seed 1977) or physical shearing (Roberts et al. 1983), but until recently, little was progressed beyond the identification of few proteins, using antibody or histochemical studies. With the advent of mass spectrometry (MS)-based proteomics, together with the recent sequencing of the $S$. mansoni genome (www.schistoDB.org) and transcriptome (Verjovski-Almeida et al. 2003), it has become possible to compile a much more complete inventory of the protein constituents of the tegument and its surface. The proteomic approach generates information on peptide sequence that can be used to search for matches to encod- 


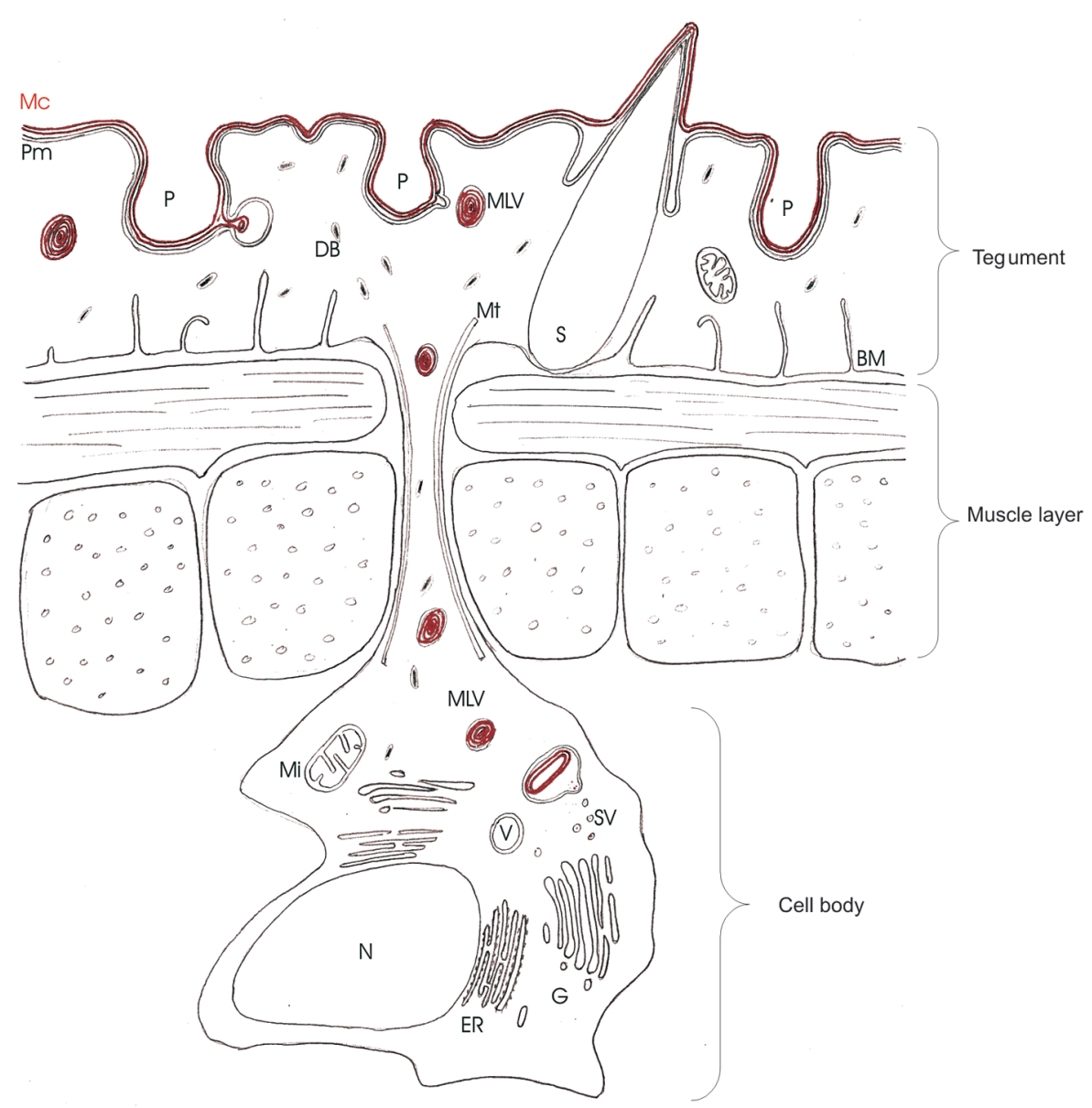

Diagrammatic representation of the Schistosoma mansoni tegument and an associated cell body (not to scale). BM: basal membrane; DB: discoid body; ER: endoplasmic reticulum; G: Golgi apparatus; Mc: membranocalyx; Mi: mitochondria; MLV: multilaminate vesicle; Mt: microtubule; N: nucleus; P: pits; Pm: plasma membrane; S: spine; SV: shuttle vesicles; V: vacuole.

ing DNA sequences in the genome and transcriptome databases. Putative functions can be assigned, based on the homology to known proteins in other organisms, while transmembrane domains, signal sequences and glycosylation sites can be predicted using specific algorithms. Several recent studies have sought to determine the protein composition of the tegument, its surface membranes and the enriched membranocalyx, and their methods and results are reviewed in this article.

\section{MS-based study of the tegument proteome}

The first proteomic analysis of the $S$. mansoni tegument utilised the freeze-thaw method developed by Roberts et al. (1983) to detach the tegument syncytium from the underlying worm body (van Balkom et al. 2005). The proteins in the whole tegument and the body fractions were separated by 1-D electrophoresis, the gel sectioned and each piece treated with trypsin. The resultant peptides were separated by reversed-phase liquid chromatography prior to tandem mass spectrometry (GeLC-MS). In all, 740 proteins were identified and assigned to three categories: tegument only; worm body only; common to both. A total of 222 proteins was found in the tegument,
43 of which were not identified in the stripped body. However, all tegument proteins must originate in the cell bodies where the synthetic machinery resides, and these cells remain in the denuded body fraction, where they constitute only a tiny proportion of the total protein. Therefore, the detection of a protein in the tegument is an indication of its abundance relative to the whole worm body, not its absence from the latter. It should be noted that the sensitivity of tandem MS makes low abundance proteins difficult to identify (discussed below). Nonetheless, the identities found in the whole tegument fraction provide valuable insights into the protein composition of this syncytium and its surface membranes (selected identities are shown in the Table, column 1).

\section{Proteomics of the tegument surface membranes}

We have used proteomics, in combination with cell fractionation techniques, to identify the proteins of the apical bilayers at the tegument surface (Braschi et al. 2006). The tegument was detached from approximately 2000 worm pairs using the freeze-thaw method, and the surface membranes were enriched by density gradient centrifugation (Roberts et al. 1983) prior to further analysis. Furthermore, 
we incorporated a differential extraction procedure on the membrane fraction with reagents of increasing solubilising strength, to remove sequentially the cytosolic and cytoskeletal proteins, yielding an insoluble pellet highly enriched in membranes. The soluble proteins were separated by $2-\mathrm{DE}$, and identities were sought after tryptic digestion of individual spots, followed by mass spectrometry. The insoluble membrane pellet was analysed by two LCbased methods. The first used GeLC-MS, as described above, while the second involved a tryptic digestion of proteins prior to peptide separation by two phases of chromatography; a strong cation exchange column was used in the first dimension and a reversed-phase column in the second. The 2-DE-based analysis enabled estimates to be made of the relative abundance of individual soluble proteins. The LC-based techniques did not provide information about relative abundance, but successfully separated peptides from hydrophobic proteins prior to their identification by MS (Table, column 2). In total, 87 proteins were identified and categorised according to their putative functions and/or sub-cellular locations.

The same approach was used with a larger starting preparation (approximately 6000 worm pairs), with LC fractionation replacing 2-DE analysis of the soluble fractions to increase detection of low abundance proteins (Braschi et al., manuscript in preparation). Approximately 200 schistosome proteins were identified as well as several host components (Table, column 3). Based on predicted properties and subcellular location of homologous proteins in other organisms, it was possible to predict the relative positions of these proteins in the schistosome tegument apex. However, no information was available to determine their accessibility to the external environment.

\section{Labelling exposed proteins on the tegument surface}

The range of proteins exposed on the surface of live adult worms was explored by covalent attachment of biotin tags that facilitated their recovery. We used two forms of sulfo-NHS-biotin, differing only in the length of their spacer arm, to label exposed lysine and N-terminal residues (Braschi \& Wilson 2006). The labelled proteins were solubilised using a stepped differential extraction procedure, captured on streptavidin-coated agarose beads, and their identities sought by GeLC-MS. All proteins labelled by the long form were also tagged by the short form (Table, column 4), but several proteins were only labelled by the latter. This enabled us to make inferences about the relative accessibility of tegument components, with those tagged exclusively by the short form reagent having a more concealed location within the membrane complex. As well as several schistosome membrane proteins, components of the mouse immune system were tagged by both reagents, suggesting an external location on the tegument.

\section{Membranocalyx enrichment}

Two distinct approaches have been developed to enrich the membranocalyx from the plasma membrane and underlying tegument syncytium (Braschi 2006). In the first, cationised ferritin (CF) was used to induce the rapid sloughing of the membranocalyx, as previously described
(Wilson \& Barnes 1977). Less than $8 \mathrm{~h}$ after a pulse labelling with $\mathrm{CF}$, the tagged membranocalyx had been completely sloughed from the surface of the tegument and replaced by a new, unlabelled layer. The released material was pelleted and the protein contents were identified by GeLC-MS (Table, column 5). In the second approach, rat anti-mouse erythrocyte ghost antibodies (RAMEGA) were used to label worms perfused from murine hosts. The tegument was then stripped using the freeze-thaw method and beads coated with goat anti-rat antibodies were used to capture the RAMEGA-labelled membranes. After thorough wash steps, the material attached to the beads was examined by electron microscopy and its protein content analysed by GeLC-MS (Table, column 6).

In both experiments, an absolute purification of the membranocalyx remained elusive, since intracellular proteins (e.g. actin, dynein light chain) were identified in the recovered samples. Nonetheless, the two approaches served to enrich the most peripheral proteins of the tegument, and the identities obtained are likely to represent molecules most closely associated with the membranocalyx, or located in the space between it and the underlying plasma membrane.

\section{Identities of the tegument surface proteins}

Selected tegument surface proteins identified by the recent proteomics studies are highlighted in the following sections and, where possible, their putative functions are discussed.

\section{Host proteins}

The ability of the schistosome to sequester molecules from its mammalian host has been known for many years. Glycolipids from the erythrocyte plasma membrane are readily transferred to the tegument surface (Goldring et al. 1976), a process that occurs even with fixed worms (Dean 1974), suggesting a passive mechanism of incorporation. Later studies revealed that host plasma proteins, including immunoglobulin (Kemp et al. 1978) and complement components (reviewed by Skelly 2004), could be demonstrated at the surface of ex-vivo worms.

In three of the proteomic studies host proteins were detected by biotin labelling and compositional analysis of the tegument surface. IgG1, IgG3, and IgM, plus a fragment of complement $\mathrm{C} 3$, were recovered after surface biotinylation (Braschi \& Wilson 2006). A further set of host proteins, including complement $\mathrm{C} 4$, a complement regulatory protein (Crry), CD44 and integrin $\alpha 2$ was identified after membrane-enrichment and LC-MS (Braschi \& Wilson, manuscript in preparation). It is notable that no mouse proteins were identified in the original study of membrane composition performed by Braschi et al. (2006), quite possibly because their concentration in the preparation was below the threshold for detection by MS. Apparently the streptavidin affinity purification of biotinylated host proteins enriches them to the point where they become detectable by proteomics. We therefore infer that, with the exception of erythrocyte glycolipids, host components are present only in trace amounts. van Balkom et al. (2005) did not find any host proteins but these workers did not record having used their MS data 
TABLE

Host and parasite proteins identified at the tegument surface

\begin{tabular}{|c|c|c|c|c|c|c|}
\hline & \multicolumn{6}{|c|}{ Study $^{a}$} \\
\hline & 1 & 2 & 3 & 4 & 5 & 6 \\
\hline \multicolumn{7}{|l|}{ Host proteins } \\
\hline IgG1 & & & & $\mathrm{S} \& \mathrm{~L}$ & $\mathrm{X}$ & \\
\hline IgG3 & & & & $\mathrm{S} \& \mathrm{~L}$ & & \\
\hline IgM & & & & $\mathrm{S} \& \mathrm{~L}$ & & \\
\hline Complement C4 & & & $\mathrm{X}$ & & & \\
\hline Complement $\mathrm{C} 3$ fragment & & & & $\mathrm{S} \& \mathrm{~L}$ & & \\
\hline Integrin alpha 2 & & & $\mathrm{X}$ & & & \\
\hline Crry complement regulatory protein & & & $\mathrm{X}$ & & & \\
\hline CD44 & & & $\mathrm{X}$ & & & \\
\hline \multicolumn{7}{|l|}{ Putative membranocalyx } \\
\hline Sm 29 & $\mathrm{~T} \& \mathrm{~B}$ & $\mathrm{X}$ & $\mathrm{X}$ & $\mathrm{S} \& \mathrm{~L}$ & $\mathrm{X}$ & $\mathrm{X}$ \\
\hline \multicolumn{7}{|l|}{ Transporters } \\
\hline SGTP4 & $\mathrm{T}$ & & $\mathrm{X}$ & & & \\
\hline SGTP1 & $\mathrm{T} \& \mathrm{~B}$ & $\mathrm{X}$ & $\mathrm{X}$ & & $\mathrm{X}$ & \\
\hline Amino acid transporter (B type) & & $\mathrm{X}$ & $\mathrm{X}$ & & & \\
\hline SNaK1 & $\mathrm{T} \& \mathrm{~B}$ & $\mathrm{X}$ & $\mathrm{X}$ & $\mathrm{S}$ & & $\mathrm{X}$ \\
\hline Copper ion transporter & & $\mathrm{X}$ & $\mathrm{X}$ & & & \\
\hline Plasma membrane calcium ATPase & $\mathrm{T} \& \mathrm{~B}$ & $\mathrm{X}$ & $\mathrm{X}$ & & & \\
\hline Aquaporin & $\mathrm{T}$ & $\mathrm{X}$ & $\mathrm{X}$ & & & \\
\hline Voltage-dependent anion channel & & $\mathrm{X}$ & $\mathrm{X}$ & $\mathrm{S}$ & & \\
\hline \multicolumn{7}{|l|}{ Membrane structural proteins } \\
\hline Annexin & $\mathrm{T} \& \mathrm{~B}$ & $\mathrm{X}$ & $\mathrm{X}$ & $\mathrm{S} \& \mathrm{~L}$ & $\mathrm{X}$ & $\mathrm{X}$ \\
\hline Dysferlin & $\mathrm{T}$ & $\mathrm{X}$ & $\mathrm{X}$ & S \& L & $\mathrm{X}$ & \\
\hline Tetraspanin A (Sj25) & & $\mathrm{X}$ & $\mathrm{X}$ & & & \\
\hline Tetraspanin B (TE736) & $\mathrm{T}$ & $\mathrm{X}$ & $\mathrm{X}$ & $\mathrm{S}$ & & \\
\hline Tetraspanin C (Sm23) & $\mathrm{B}$ & $\mathrm{X}$ & $\mathrm{X}$ & & $\mathrm{X}$ & \\
\hline Tetraspanin D (CD63-like TSP-2) & $\mathrm{T}$ & & $\mathrm{X}$ & $\mathrm{S} \& \mathrm{~L}$ & $\mathrm{X}$ & $\mathrm{X}$ \\
\hline Tetraspanin E (unknown) & & & $\mathrm{X}$ & $\mathrm{S} \& \mathrm{~L}$ & & \\
\hline Multidrug resistance protein 2 & $\mathrm{~B}$ & & $\mathrm{X}$ & & & \\
\hline Scramblase & & & $\mathrm{X}$ & & & \\
\hline Phospholipid transporting ATPase & & & $\mathrm{X}$ & & & \\
\hline \multicolumn{7}{|l|}{ Membrane enzymes } \\
\hline Phosphodiesterase 5 & $\mathrm{~T}$ & $\mathrm{X}$ & $\mathrm{X}$ & $\mathrm{S} \& \mathrm{~L}$ & & \\
\hline Diphosphohydrolase & $\mathrm{T}$ & $\mathrm{X}$ & $\mathrm{X}$ & S \& L & & \\
\hline Alkaline phosphatase & $\mathrm{T} \& \mathrm{~B}$ & $\mathrm{X}$ & $\mathrm{X}$ & $\mathrm{S} \& \mathrm{~L}$ & & \\
\hline Acetylcholinesterase & & & $\mathrm{X}$ & & & \\
\hline Carbonic anhydrase & $\mathrm{B}$ & & $\mathrm{X}$ & $\mathrm{S}$ & & \\
\hline Calpain & $\mathrm{T} \& \mathrm{~B}$ & $\mathrm{X}$ & $X$ & $\mathrm{~S}$ & $\mathrm{X}$ & $\mathrm{X}$ \\
\hline \multicolumn{7}{|l|}{ Others } \\
\hline $200 \mathrm{kDa}$ surface protein & $\mathrm{T} \& \mathrm{~B}$ & & $X$ & $\mathrm{~S}$ & $\mathrm{X}$ & \\
\hline Gp18-22 (Sm25) & $\mathrm{T}$ & & $\mathrm{X}$ & & & $\mathrm{X}$ \\
\hline $\mathrm{Sm} 22.6$ & $\mathrm{~T} \& \mathrm{~B}$ & $\mathrm{X}$ & $\mathrm{X}$ & & $\mathrm{X}$ & $\mathrm{X}$ \\
\hline Paramyosin & $\mathrm{T} \& \mathrm{~B}$ & & & & & \\
\hline Actin & $\mathrm{T} \& \mathrm{~B}$ & $\mathrm{X}$ & $\mathrm{X}$ & $\mathrm{S}$ & $\mathrm{X}$ & $X$ \\
\hline
\end{tabular}

$a$ : study in which the protein was identified: (1) tegument (T) and stripped body (B) proteome (van Balkom et al. 2005); (2) surface membrane preparation (Braschi et al. 2006); (3) LC-based analysis of surface membrane preparation (Braschi \& Wilson, manuscript in preparation); (4) surface biotinylation (Braschi \& Wilson 2006), S = labeled by short form reagent, $\mathrm{L}=$ long form reagent, $\mathrm{S} \& \mathrm{~L}$ = both; (5) membranocalyx enrichment using cationised ferritin (Braschi 2006); (6) membranocalyx enrichment using affinity beads (Braschi 2006).

to search the appropriate databases.

The mouse immunoglobulins identified at the surface of the tegument may be present as a result of specific antigen binding, non-specific binding, or attachment via Fc receptors. No proteins with homology to known Fc receptors were found in any of the surface membrane preparations, but this does not rule out unique schistosome proteins performing the function. The presence of complement components (Braschi \& Wilson 2006, Braschi $\&$ Wilson, manuscript in preparation) argues that the antibodies are bound to specific antigens via their Fab region, leaving their Fc domains free to fix complement (of course, fixation of complement by the alternative or lectin binding pathways cannot be ruled out). No proteins of the terminal membrane attack complex (C5-C9) were identified, suggesting that schistosomes deploy effective in- 
hibitory mechanisms to interrupt the pathway at an early stage. Indeed, the detection of a fragment of $\mathrm{C} 3$ provides evidence for complement deactivation (Braschi \& Wilson 2006) implying that the complement attack pathway has been inhibited by specific immunoregulatory proteins. Furthermore, the identification of mouse Crry on the schistosome surface (Braschi \& Wilson, manuscript in preparation), as well as previous reports of other acquired complement regulatory proteins, such as decay accelerating factor (Pearce et al. 1990), suggest that the parasite is able to utilise host proteins for this purpose.

It is difficult to envisage how membrane-spanning proteins such as CD44 and integrin $\alpha 2$ transfer from a host cell to the schistosome surface, because they possess complete transmembrane domains (in comparison with the short acyl chains of glycolipids and GPI-anchored proteins). The simplest explanation is that a close apposition between the extremely hydrophobic surface of the membranocalyx and host cells, results in the "flipping" of proteins from one bilayer to the other. Alternative hypotheses include the fusion of host plasma membranes with the membranocalyx (Caulfield et al. 1980), or the acquisition of "blebs" from host cells (Pearce et al. 1990). Similarly, exosomes released from blood or endothelial cells (Pilzer et al. 2005) might readily fuse with the lipophilic tegument surface, rather like a bubble landing in soapy water. As exosomes represent small patches of plasma membrane from their parent cell, sometimes enriched in specific proteins (Butikofer et al. 1989), their incorporation into the membranocalyx would add both lipids and membrane proteins. The concentration of membrane-spanning proteins appears too low to provide much of a disguise for the parasite and may simply be the result of intimate contact with endothelia during intravascular movement. However, the acquisition of complement regulatory proteins, even at low concentrations could provide the parasite with protection from complement-mediated attack. In support of such an hypothesis, exosomes released from dendritic and B cells were shown to express CD55 and CD59 and were protected from complementmediated lysis (Clayton et al. 2003). Of course, whether or not the host proteins acquired by schistosomes are still fully operational cannot be determined by MS-based proteomic studies, and their role (if any) at the schistosome surface will only be elucidated by functional studies.

\section{Putative membranocalyx proteins}

In contrast to the variety of host proteins identified on/in the membranocalyx, very few schistosome proteins can be confidently assigned to this outer bilayer. The membranocalyx is assembled in the lumen of the multilaminate vesicles and exocytosed at the tegument surface. It therefore does not originate from the membranes of the ER and is unlikely to contain transmembrane or GPI-anchored proteins when formed. This means that only proteins with a signal sequence, lacking an anchor in the ER membrane are likely to pass into the lumen of the multilaminate vesicles and associate with their lipid contents. Using algorithms to search for putative signal sequences and transmembrane domains it was postulated that Sm29 might represent a true component of the membranocalyx (Braschi et al. 2006, Braschi \& Wilson 2006). By inference, we conclude that the membrane-spanning and GPI-anchored proteins are located within, or on the surface of, the plasma membrane. These new data support the original hypothesis (Wilson \& Barnes 1974a) that the membranocalyx is a lipid-rich membrane-like secretion that serves as a physical barrier to protect the vulnerable underlying plasma membrane.

\section{Nutrient transporters at the tegument surface}

Although the parasite obtains most of its dietary requirements by the ingestion of erythrocytes, it is clear the tegument provides a significant source of nutrients and ions, a system upon which the worm may rely during its migratory stages through the mammalian host. The extensive folded and pitted surface of the tegument is consistent with an absorptive function, and it is unsurprising that the schistosome takes advantage of the high concentration of newly-absorbed nutrients in the hepatic portal system of its host. Transporters of sugars (SGTP1 and SGTP4) and amino acids (B type) were identified by proteomics (Table). Regulated ion movement across the plasma membrane was also evident, with calcium, sodium, potassium, copper, and anion transporting proteins identified. The presence of aquaporins indicates the flux of water and solutes across the plasma membrane. The uptake of calcium appears crucial for the operation of many schistosome proteins, such as calpain and annexin, and this ion may play an integral role in the functioning of the syncytium. Indeed, the antischistosomal drug, praziquantel, is thought to disrupt calcium homeostasis, which damages the tegument (reviewed by Greenberg 2005).

\section{Tetraspanins and membrane structural proteins}

The identification of five tetraspanins in the various surface membrane preparations points to these proteins as abundant components at the tegument periphery. By analogy with other organisms, we speculate that schistosome tetraspanins play a structural role in the plasma membrane (Levy \& Shoham 2005b). Their capacity for homologous interaction to form a tetraspanin web (Levy \& Shoham 2005a) may provide an organized scaffold within the lipid bilayer upon which other proteins assemble. Similarly, their extracellular loops may provide platforms for proteins or glycans that interact with the membranocalyx. That some tetraspanins are identified more readily than others, and only three biotinylated, suggests that they are present at differing concentrations or locations within the tegument surface complex. Again, their precise role at the schistosome periphery will only be elucidated by functional studies.

Three other proteins, annexin, dysferlin and calpain were reproducibly identified in proteomic analyses of the tegument surface. The phospholipid-binding properties of annexin (Rescher \& Gerke 2004), together with its identification in membranocalyx-enrichment experiments (Braschi 2006) make this protein an ideal candidate to mediate the attachment of the membranocalyx to the underlying plasma membrane. Dysferlin, by analogy with its homologues in other organisms, may play a key role in 
membrane repair and/or vesicle fusion at the tegument surface (Bansal \& Campbell 2004). Similarly, we speculate that calpain is a vital tool for the remodeling of the cytoskeleton that lies directly beneath the plasma membrane, and this protease may also play a role in the fusion of the multilaminate vesicles (Molinari \& Carafoli 1997).

The identification of proteins that function in the (a-) symmetrical distribution of phospholipids within a bilayer, such as scramblase, phospholipid transporting ATPase and multidrug resistance protein 2 (MDR2), provides some pointers to the lipid dynamics within the tegument. The formation of the multilaminate vesicles within the tegument cell body is not fully understood, but it is possible that these proteins are involved, as follows. If $S$. mansoni MDR2 were present in the bounding membrane of the multilaminate vesicle and functioned in a similar way to its homologue in mammals, i.e. flipping phospholipids from the cytoplasmic to the extracellular face of plasma membrane in hepatocytes (Smit et al. 1993), it could create an excess concentration of phospholipids in the luminal face of the multilaminate vesicle. Such a situation would promote phospholipids to enter the lumen of the vesicle, in a similar manner to phospholipid entry into the bile duct (Smit et al. 1993). In the aqueous environment of the vesicle lumen, we assume that bipolar lipids would self-assemble into the characteristic concentric circles, preventing the interaction of water with their hydrophobic tails.

\section{Membrane bound enzymes}

A group of three phosphate hydrolysing proteins, alkaline phosphatase, phosphodiesterase, and diphosphohydrolase, were biotinylated by the long form reagent, demonstrating the accessibility of their large extracellular domains (Braschi \& Wilson 2006). Presumably, these enzymes function in a similar way to their homologues in other eukaryotic species by removing phosphate groups from organic molecules to facilitate the passage of both through the plasma membrane. Thus, the presence of their catalytic domains external to the plasma membrane but shielded by the membranocalyx, requires that pores must exist in the latter to allow the ingress of substrate (and the biotinylation reagents).

The proposed barrier properties of the membranocalyx would also appear to be an obstacle to the uptake of nutrients and solutes from the bloodstream. Given our supposition that classical membrane transport proteins are absent from the membranocalyx, some other mechanism must allow the ingress of small molecules, potentially a non-conventional pore-forming structure. An alternative, such as beta barrel proteins found in the outer membrane of gram negative bacteria would fit the bill, but we have yet to identify such structures in our tegument preparations.

Carbonic anhydrase, an enzyme that catalyses $\mathrm{CO}_{2}+$ $\mathrm{H}_{2} \mathrm{O} \leftrightarrow \mathrm{HCO}_{3}^{-}+\mathrm{H}^{+}$in the cytoplasm of erythrocytes (Reithmeier 2001), has been identified in proteomic analyses of the tegument surface. The schistosome homologue has a putative GPI-anchor, and its catalytic domain is therefore likely to be external to the plasma membrane. The role of this enzyme outside the tegument syncytium is unknown, but it might function to hydrate $\mathrm{CO}_{2}$ released by the parasite during respiration. Although the $S$. mansoni transcriptome encodes a $\mathrm{HCO}_{3}{ }^{-} / \mathrm{Cl}^{-}$transporting protein homologous to that found in mammalian erythrocytes (accession no. Sm07207), it has not been identified by proteomics. However, other anion channels have been found which may be responsible for the flux of $\mathrm{Cl}^{-}$ions in/ out of the tegument.

\section{Schistosome-specific proteins}

Perhaps the greatest accomplishment of the proteomic studies on the $S$. mansoni tegument is the identification of numerous proteins that are unique to the parasite. As stated by van Balkom et al. (2005), these proteins may perform roles that are specific to the schistosome tegument, with their unique sequence and structure making them excellent targets for anti-schistosome drugs. Approximately $27 \%$ of all the MS matches to EST or genomic sequences in the $S$. mansoni database shared no homology to any entries in the NCBInr database (Braschi \& Wilson, manuscript in preparation). When we used smaller amounts of starting material (Braschi et al. 2006, Braschi $\&$ Wilson 2006), the percentage of unmatched proteins was significantly lower ( $18 \%$ or less). Thus, we surmise that the abundant tegument proteins are the ones most likely to have been characterized. The fact that approximately $55 \%$ of $S$. mansoni genes have no assignable function (Verjovski-Almeida et al. 2003) suggests that only when this percentage is attained in proteomic analysis, will we have reached complete coverage. This means that we are still some way off the total characterization of the tegument surface proteome. A number of proteins that have been postulated as integral to the schistosome surface were not identified by proteomics. The failure to detect them by MS-based studies, whilst not ruling out their presence, suggests that they are not major constituents.

It is ironic that in spite of their ability to persist in the host blood stream for decades, schistosomes are very fragile in vitro and need to be manipulated with great care. Thus, studies such as we have undertaken should begin with worms perfused from mice in a culture medium such as RPMI 1640 rather than with the commonly advocated citrate saline. Similarly, worms should never be handled with tweezers but only transferred with wide-bore Pasteur pipettes. Lastly, they deteriorate rapidly in vitro unless protein supplements are present, so incubations of more than $4 \mathrm{~h}$ in serum-free culture medium should be avoided (Wilson \& Barnes 1974b). Failure to take such precautions will inevitably lead to worm damage and generate misleading results. In this context, it is evident from the differential extraction of enriched tegument surface membranes (Braschi et al. 2006) that a number of components previously described as exposed on the tegument surface are readily detached. These include proteins such as $\mathrm{Sm} 22.6, \mathrm{Sm} 21.7$ and dynein light chain, which we believe contribute to the cytoskeleton lying immediately beneath the plasma membrane and are responsible for maintenance of the pitted tegument surface architecture.

\section{Conclusion}

The recent proteomic studies have provided a wealth of data on the protein constituents of the schistosome 
tegument and their relative locations within this surface complex. Future studies with more refined techniques to isolate the surface layers and enrich for particular classes of constituent, coupled with an increased sensitivity of MS detection, will undoubtedly add further proteins to our list (the absence of receptors is especially notable). Exploring the function of specific components such as annexin or Sm29 would be greatly enhanced if it were possible to disrupt their encoding genes by technologies such as RNA interference.

\section{REFERENCES}

Bansal D, Campbell KP 2004. Dysferlin and the plasma membrane repair in muscular dystrophy. Trends Cell Biol 14: 206-213.

Bennett JL, Seed JL 1977. Characterization and isolation of concanavalin A binding sites from the epidermis of $S$. mansoni. J Parasitol 63: 250-258.

Braschi S 2006. Proteomic Analysis of the Schistosoma mansoni Surface Membranes, PhD thesis University of York, York.

Braschi S, Curwen RS, Ashton PD, Verjovski-Almeida S, Wilson A 2006. The tegument surface membranes of the human blood parasite Schistosoma mansoni: a proteomic analysis after differential extraction. Proteomics 6: 1471-1482.

Braschi S, Wilson RA 2006. Proteins exposed at the adult schistosome surface revealed by biotinylation. Mol Cell Proteomics 5: 347-356.

Butikofer P, Kuypers FA, Xu CM, Chiu DT, Lubin B 1989. Enrichment of two glycosyl-phosphatidylinositol-anchored proteins, acetylcholinesterase and decay accelerating factor, in vesicles released from human red blood cells. Blood 74: 1481-1485.

Caulfield JP, Korman G, Butterworth AE, Hogan M, David JR 1980. The adherence of human neutrophils and eosinophils to schistosomula: evidence for membrane fusion between cells and parasites. $J$ Cell Biol 86: 46-63.

Clayton A, Harris CL, Court J, Mason MD, Morgan BP 2003. Antigen-presenting cell exosomes are protected from complement-mediated lysis by expression of CD55 and CD59. Eur J Immunol 33: 522-531.

Dean DA 1974. Schistosoma mansoni: adsorption of human blood group A and B antigens by schistosomula. J Parasitol 60: 260-263.

Goldring OL, Clegg JA, Smithers SR, Terry RJ 1976. Acquisition of human blood group antigens by Schistosoma mansoni. Clin Exp Immunol 26: 181-187.

Greenberg RM 2005. Are Ca2+ channels targets of praziquantel action? Int J Parasitol 35: 1-9.

Harris AR, Russell RJ, Charters AD 1984. A review of schistosomiasis in immigrants in Western Australia, demonstrating the unusual longevity of Schistosoma mansoni. Trans $R$ Soc Trop Med Hyg 78: 385-388.

Hockley DJ, McLaren DJ 1973. Schistosoma mansoni: changes in the outer membrane of the tegument during development from cercaria to adult worm. Int J Parasitol 3: 13-25.

Kemp WM, Merritt SC, Rosier JG 1978. Schistosoma mansoni: identification of immunoglobulins associated with the tegument of adult parasites from mice. Exp Parasitol 45: 81-87.
Kusel JR 1970. Studies on the surfaces of cercariae and schistosomula of Schistosoma mansoni. Parasitology 61: 127-134.

Levy S, Shoham T 2005a. The tetraspanin web modulates immune-signalling complexes. Nat Rev Immunol 5: 136-148.

Levy S, Shoham T 2005b. Protein-protein interactions in the tetraspanin web. Physiology (Bethesda) 20: 218-224.

McDiarmid SS, Dean LL, Podesta RB 1983. Sequential removal of outer bilayer and apical plasma membrane from the surface epithelial syncytium of Schistosoma mansoni. Mol Biochem Parasitol 7: 141-157.

Molinari M, Carafoli E 1997. Calpain: a cytosolic proteinase active at the membranes. J Membr Biol 156: 1-8.

Oaks JA, Cain GD, Mower DA, Raj RK 1981. Disruption and removal of the tegument from Schistosoma mansoni with triton X-100. J Parasitol 67: 761-775.

Pearce EJ, Hall BF, Sher A 1990. Host-specific evasion of the alternative complement pathway by schistosomes correlates with the presence of a phospholipase C-sensitive surface molecule resembling human decay accelerating factor. J Immunol 144: 2751-2756.

Pilzer D, Gasser O, Moskovich O, Schifferli JA, Fishelson Z 2005. Emission of membrane vesicles: roles in complement resistance, immunity and cancer. Springer Semin Immunopathol 27: 375-387.

Reithmeier RA 2001. A membrane metabolon linking carbonic anhydrase with chloride/bicarbonate anion exchangers. Blood Cells Mol Dis 27: 85-89.

Rescher U, Gerke V 2004. Annexins-unique membrane binding proteins with diverse functions. J Cell Sci 117: 2631-2639.

Roberts SM, MacGregor AN, Vojvodic M, Wells E, Crabtree JE, Wilson RA 1983. Tegument surface membranes of adult Schistosoma mansoni: development of a method for their isolation. Mol Biochem Parasitol 9: 105-127.

Skelly PJ 2004. Intravascular schistosomes and complement. Trends Parasitol 20: 370-374.

Smit JJ, Schinkel AH, Oude Elferink RP, Groen AK, Wagenaar E, van Deemter L, Mol CA, Ottenhoff R, van der Lugt NM, van Roon MA et al. 1993. Homozygous disruption of the murine mdr2 P-glycoprotein gene leads to a complete absence of phospholipid from bile and to liver disease. Cell 75: 451-462.

van Balkom BW, van Gestel RA, Brouwers JF, Krijgsveld J, Tielens AG, Heck AJ, van Hellemond JJ 2005. Mass spectrometric analysis of the Schistosoma mansoni tegumental sub-proteome. J Proteome Res 4: 958-966.

Verjovski-Almeida S, DeMarco R, Martins EA, Guimaraes PE, Ojopi EP, Paquola AC, Piazza JP, Nishiyama MY, Jr., Kitajima JP, Adamson RE, Ashton PD, Bonaldo MF, Coulson PS, Dillon GP, Farias LP, Gregorio SP, Ho PL, Leite RA, Malaquias LC, Marques RC, Miyasato PA, Nascimento AL, Ohlweiler FP, Reis EM, Ribeiro MA, Sa RG, Stukart GC, Soares MB, Gargioni C, Kawano T, Rodrigues V, Madeira AM, Wilson RA, Menck CF, Setubal JC, Leite LC, Dias-Neto E 2003. Transcriptome analysis of the acoelomate human parasite Schistosoma mansoni. Nat Genet 35: 148-157.

Wilson RA, Barnes PE 1974a. The tegument of Schistosoma 
mansoni: observations on the formation, structure and composition of cytoplasmic inclusions in relation to tegument function. Parasitology 68: 239-258.

Wilson RA, Barnes PE 1974b. An in vitro investigation of dynamic processes occurring in the schistosome tegument, using compounds known to disrupt secretory processes. Parasitology 68: 259-270.

Wilson RA, Barnes PE 1977. The formation and turnover of the membranocalyx on the tegument of Schistosoma mansoni. Parasitology 74: 61-71. 